\title{
Which orthodontic adhesive should I use? - no clear answer yet
}

\author{
What is the most effective adhesive when bonding orthodontic brackets to \\ teeth?
}

\begin{abstract}
Mandall NA, Millett DT, Mattick CR, Hickman J, Macfarlane TV, Worthington HV. Adhesives for fixed orthodontic brackets (Cochrane Review). The Cochrane Library 2003; Issue 2. Oxford: Update Software
\end{abstract}

Data sources The Cochrane Oral Health Group's Trials Register, the Cochrane Central Register of Controlled Trials, Medline and EMbase, up to August 2002, were used to identify publications.

Study selection Selected studies were randomised controlled trials (RCT) and controlled clinical trials that compared two different adhesives in subjects who required fixed orthodontic appliances and where the intervention was adhesive bonding of stainless steel brackets to all teeth except the molars. The primary outcome was debond or bracket failure.

Data extraction and synthesis Data were recorded for decalcification as a secondary outcome, if present. Information regarding methods, participants, interventions, outcome measures and results were extracted in duplicate by pairs of reviewers. Since the data were not amenable to meta-analysis, the results are presented in narrative form only.

Results Three trials satisfied the inclusion criteria. A chemically-cured composite was compared with a light-cure composite (one trial), a conventional glass ionomer cement (GIC; one trial) and a polyacidmodified resin composite (compomer; one trial). The quality of the trial reports was generally poor.

Conclusions It is not possible to draw anything other than tentative conclusions from this systematic review of orthodontic adhesives, primarily because of the weakness in the design and reporting of existing trials. Therefore, at present there is no clear evidence with which to make a clinical decision on the type of orthodontic adhesive to use. A number of suggestions are made for methods of improving future research involving orthodontic adhesives.

\section{Commentary}

The advent of adhesive dentistry and direct bonding of orthodontic brackets is a dramatic event that has changed the course of clinical orthodontics. The product development and technological advances are so rapid that it is difficult for a clinician to remain oriented, and the conflicting claims of product superiority from manufacturers further complicates the issue. This systematic review therefore addresses a pressing concern for the clinician. The authors should be complimented on focusing on such a contemporary issue.

The review has been structured with precision and detail with regard to evidence-based methods. It highlights the large number of clinical trials of orthodontic adhesives which largely compare chemical-cure composites with either light-cured or chemical-cure GIC cements.

The definition of quality and its assessment is most impressive, with two reviewers weighing the quality of each paper and an

Address for correspondence: Emma Tavender, Review Group Co-ordinator, Cochran Oral Health Group, University Dental Hospital of Manchester, Higher Cambridge Street, Manchester M15 6FH, UK. E-mail: emma.tavender@man.ac.uk independent third assessment in the event of a discrepancy. The reviewers have surgically explored each trial for evidence and it is therefore not surprising that only three trials fit the inclusion criteria. Even the check on the methodological quality of the two included trials shows disputed areas in selection, detection, attrition and performance bias. Again, it is not surprising that the results of the review are therefore inconclusive. A synthesis of data and a meta-analysis was not possible. It only highlights the frustration at inadequacy of acceptable scientific evidence in orthodontics.

Perhaps it would be pertinent, however, to review orthodontic adhesives more broadly. The issue of orthodontic bonding has perhaps been oversimplified in addressing only: metal brackets and debonding, and enamel demineralisation. It is an accepted fact that the bracket profiles, the material (ceramic/metal) and the base (laser/mesh) all alter the bonding profile substantially. ${ }^{1}$

A statement that at present there is no evidence with which to decide on the best adhesive type for use needs to be balanced. There are distinct clinical advantages of light-cured materials over chemically-cured adhesives. A current $\mathrm{RCT}^{2}$ only endorses the issue that while chemically cured composites show better bond strength, this is usually $24 \mathrm{~h}$ later. While light cured composites which fall within the range of acceptable clinical bond strength would permit immediate wire placement and loading of brackets - in itself a great clinical advantage.

The reviewers are absolutely correct in concluding that there is no strong evidence to answer the basic issue of which is the best adhesive, but perhaps further work on this issue would take into account the variability in brackets, the clinical advantages of various materials such as light-cured, wet-field adhesives and the increasing proportion of adult patients in whom greater masticatory stresses impose additional demands on materials. The review succeeds in highlighting the glaring inadequacy of scientific evidence and certainly provides a direction towards improving the quality of future research.

\section{Practice point}

- There is no strong evidence to answer the basic issue of which is the best adhesive for orthodontic brackets.

\section{Anmol S Kalha}

Director and Cordinator, Centre for Evidence Based Dentistry, College of Dental Sciences, Davangere, Karnataka, India

1. Sorel O, El Alam R, Chagneau F, Cathelineau G. Comparison of bond strength between simple foil mesh and laser-structured base retention brackets. Am J Orthod Dentofacial Orthop 2002; 122:260-622.

2. Toledano M, Osorio R, Osorio E, Romeo A, de la Higuera B, Garcia-Godoy. Bond strength of orthodontic brackets using different light and self-curing cements. Angle Orthod 2003; 56-63.

3. Fox NA, McCabe JF, Buckley JG. A critique of bond strength testing in orthodontics. $\mathrm{Br}$ J Orthod 1994; 33-43.

Evidence-Based Dentistry (2003) 4, 52.

doi:10.1038/sj.ebd.6400194 